\title{
Teil der Community
}

Geht Ihnen das auch so? Spätestens nach der Registrierung für eine Fortbildung steigt die Spannung, welchen neuen oder bekannten Gesichtern man begegnen wird. Themen aus der Praxis mit Kollegen besprechen, Rat und Impulse einholen und sich über den neuesten Klatsch aus der Ergoszene austauschen. Das sind für mich die i-Tüpfelchen von Veranstaltungen.

Auf einem solch herrlichen Nebenschauplatz befand ich mich kürzlich im Rahmen der Fachtagung in Graz. Im Vorfeld hatte ich mich mit einer mir bis dahin unbekannten Ergotherapeutin verabredet - wir sind uns lediglich mal auf Facebook „über den Weg gelaufen“. Im vereinbarten Café erkannten wir uns glücklicherweise auf Anhieb, bestellten freudestrahlend Cappuccino, und dann gab es kein Halten mehr: Wir plauderten und schwärmten zwei Stunden lang über die Ergotherapie, als würden wir uns seit Jahrzehnten kennen. Den Rückflug trat ich mit jeder Menge Erkenntnisse und Ideen im Gepäck an.
Menschen zu treffen, mit denen man auf einer Wellenlänge liegt, die verstehen, wovon man redet, und die über dieselben InsiderWitze lachen können, das schätze ich an der Gemeinde Ergotherapie. Raum für diesen Community-Gedanken möchten wir Ihnen $a b$ sofort auch in jeder Ausgabe von ergopraxis geben. Dazu haben wir unsere Rubrik "Leserbriefe" aufgepimpt. Sie heißt jetzt „Community“ (○ SEITE 6). In dieser Rubrik freuen wir uns nach wie vor über Ihre Leserbriefe, Lob und Tadel, aber auch über Tipps zu Veranstaltungen, sehenswerten TV-Sendungen oder Alltagstricks, die Sie mit Ihren Kollegen teilen möchten. Hier ist auch ganz viel Platz zum Schmunzeln: Falls Ihnen Lustiges aus den sozialen Medien über den Weg läuft - immer her damit!

\section{Ihr Gemeindemitglied}

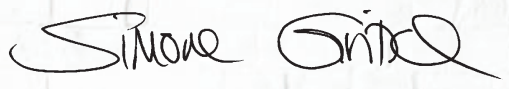

